\title{
Fucosylated C4b-binding protein $\alpha$-chain, a novel serum biomarker that predicts lymph node metastasis in pancreatic ductal adenocarcinoma
}

\author{
KAZUYUKI SOGAWA $^{1}$, SAKINO YAMANAKA ${ }^{1}$, SHIGETSUGU TAKANO ${ }^{2}$, KOSUKE SASAKI ${ }^{2}$, \\ YOJI MIYAHARA ${ }^{2}$, KATSUNORI FURUKAWA ${ }^{2}$, TSUKASA TAKAYASHIKI ${ }^{2}$, SATOSHI KUBOKI ${ }^{2}$, \\ HIROTAKA TAKIZAWA ${ }^{3}$, FUMIO NOMURA ${ }^{4}$ and MASAYUKI OHTSUKA ${ }^{2}$ \\ ${ }^{1}$ Department of Biochemistry, School of Life and Environmental Science, Azabu University, Sagamihara, \\ Kanagawa 252-5201; ${ }^{2}$ Department of General Surgery, Graduate School of Medicine, Chiba University, \\ Chiba 260-8670; ${ }^{3}$ Kashiwado Clinic in Port-Square, Kashiwado Memorial Foundation, Chiba 260-0025; ${ }^{4}$ Divisions \\ of Clinical Mass Spectrometry and Clinical Genetics, Chiba University Hospital, Chiba 260-8670, Japan
}

Received July 19, 2020; Accepted November 5, 2020

DOI: $10.3892 / \mathrm{ol} .2020 .12388$

\begin{abstract}
C4b-binding protein $\alpha$-chain (C4BPA) was previously identified as a novel serum biomarker for pancreatic ductal adenocarcinoma (PDAC). To apply this biomarker for clinical diagnosis, a lectin ELISA was established to measure serum fucosylated (Fuc)-C4BPA levels in 45 patients with PDAC, 20 patients with chronic pancreatitis (CP) and 50 healthy volunteers (HVs) in one training and three validation sets. The lecithin ELISA developed in the current study exhibited satisfactory within-run (2.6-6.7\%) and between-day (1.8-3.6\%) coefficient of variations. Serum Fuc-C4BPA levels in patients with PDAC $(0.54 \pm 0.27 \mathrm{AU} / \mathrm{ml})$ was significantly higher than that in HVs $(0.21 \pm 0.06 \mathrm{AU} / \mathrm{ml} ; \mathrm{P}<0.0001)$ and patients with $\mathrm{CP}(0.25 \pm 0.03 \mathrm{AU} / \mathrm{ml} ; \mathrm{P}<0.0001)$. Additionally, serum Fuc-C4BPA levels in preoperative patients were significantly decreased compared with postoperative patient sera $(\mathrm{P}<0.0003)$. The receiver operating characteristic $(\mathrm{ROC})$ curve analyses revealed that the area under the curve (AUC) of Fuc-C4BPA (0.985) was higher than that of carbohydrate antigen (CA)19-9 (0.843), carcinoembryonic antigen (0.548) and total C4BPA $(0.875)(\mathrm{P}<0.001)$. To analyze the clinical significance of Fuc-C4BPA, the ability of Fuc-C4BPA to predict lymph node metastasis was compared with that of CA19-9. The AUC of serum Fuc-C4BPA levels (0.703) was significantly higher than that of serum CA19-9 levels (0.500) in patients with PDAC $(\mathrm{P}<0.001)$. The current study established
\end{abstract}

Correspondence to: Dr Shigetsugu Takano, Department of General Surgery, Graduate School of Medicine, Chiba University, 1-8-1 Inohana, Chuo-ku, Chiba 260-8670, Japan

E-mail: stakano@faculty.chiba-u.jp

Key words: fucosylated C4-binding protein $\alpha$-chain, lectin enzyme-linked immunosorbent assay, pancreatic ductal adenocarcinoma, serum biomarker, lymph node metastasis a novel lectin ELISA for measuring serum Fuc-C4BPA levels. Thus, Fuc-C4BPA has potential clinical applications owing to its high diagnostic value in PDAC.

\section{Introduction}

Pancreatic ductal adenocarcinoma (PDAC), a malignant tumor, is associated with poor prognosis, which is mainly due to the difficulties in early diagnosis and the low efficacy of adjuvant therapy. There is a need to develop methods for early diagnosis and multimodal treatment involving surgical resection and chemo-radiotherapy to improve the clinical outcomes of patients with PDAC. The development of mass spectrometry (MS)-based proteomics technology has facilitated the detection of disease biomarkers (1). This technology, which involves the analysis of peptides and proteins in biological samples, has enabled the identification of many biomarker candidates in the human plasma or serum and can provide cancer-specific diagnostic information $(2,3)$.

A comprehensive analysis of glycoproteins in the serum samples of patients with PDAC using Tandem Mass $\mathrm{Tag}^{\mathrm{TM}}$ with MS identified C4b-binding protein $\alpha$-chain (C4BPA) as a novel serum PDAC biomarker (4). C4BPA is reported to be markedly upregulated in patients with clear-cell ovarian cancer (5) and non-small-cell lung cancer (6). This indicated the diagnostic value of serum C4BPA in different cancers. However, the objective comparison of serum C4BPA levels is difficult because of the use of different measurement methods. High reproducibility and specificity are the key parameters for establishing useful clinical biomarkers, large-scale validation of these markers, and high-throughput screening for such markers.

Fucosylation and sialylation are major glycosylation events in post-translational protein modifications, which are involved in the pathogenesis of inflammation and cancer (7). In fucosylation, the transfer of fucose from GDP-fucose to a molecule is catalyzed by fucosyltransferases. Sialylation involves the addition of a sialic acid unit to the end of an oligosaccharide chain 
in a glycoprotein. The glycoproteins, alpha-fetoprotein-L3 (AFP-L3; fucosylated AFP) and carbohydrate antigen 19-9 (CA19-9; sialyl Lewis A antigen) are used as tumor markers in hepatocellular carcinoma (8) and PDAC (9), respectively. Thus, glycoproteins are potential serum diagnostic biomarkers for cancer. Previous studies have reported that the fully-sialylated C4BPA levels are upregulated in the serum of patients with ovarian cancer (10). However, there are no studies on the importance of serum fucosylated (Fuc-) C4BPA in patients with cancer. Therefore, we hypothesized that the serum Fuc-C4BPA level has diagnostic and clinical significance in patients with PDAC.

In this study, we established a novel hybrid enzyme-linked immunosorbent assay (ELISA) for measuring Fuc-C4BPA using lens culinaris agglutinin (LCA)-lectin, which specifically binds to fucose. We demonstrated that serum Fuc-C4BPA is a potential diagnostic biomarker for PDAC with clinical significance.

\section{Materials and methods}

Serum samples of participants. The inclusion criterion for the study was patients aged 20-85 who have been histologically diagnosed with PDAC or chronic pancreatitis (CP). Patients with other malignancies in the active phase were excluded from the study. For the training set, we measured the serum Fuc-C4BPA level in 19 patients with PDAC, 10 patients with CP, and 40 age and gender-matched healthy volunteers (HVs). For the validation set 1 , the Fuc-C4BPA levels were comparatively analyzed in nine pairs of pre- and post-operative (3-4 weeks post-surgery when the serum levels of C-reactive protein returned to the normal range) sera obtained from patients with PDAC who underwent curative surgery. Additionally, the diagnostic values of conventional tumor markers, such as CA19-9, carcinoembryonic antigen (CEA), total C4BPA, and Fuc-C4BPA were investigated in the validation set 2 . In the validation set 3 , the serum Fuc-C4BPA levels were comparatively analyzed among $50 \mathrm{HVs}, 20$ patients with $\mathrm{CP}$, and 45 patients with PDAC to examine the correlation between the serum C4BPA levels and the clinicopathological features of patients with PDAC (Table I). The blood samples from patients with PDAC or CP were collected at the Department of General Surgery, Chiba University Hospital between May 2011 and March 2019. The samples from HVs were collected at the Kashiwado Hospital. The procedures for sample collection and processing were performed as previously reported (11). The ethics committee of each institute approved the protocol (approval number: 2155 for Chiba University, and 007 for Kashiwado Hospital). Written informed consent was obtained from all the patients and HVs. All data from the participants were fully anonymized, and the study was performed according to the guidelines of the Declaration of Helsinki 1975.

Western blot analysis. To examine the specificity of the anti-C4BPA antibody, recombinant human C4BPA protein (Abnova) and serum sample of a patient with PDAC were subjected to sodium dodecyl sulfate-polyacrylamide gel electrophoresis using a 10-20\% gradient gel (DRC, Tokyo, Japan) in the absence of $\beta$-mercaptoethanol. The resolved proteins were transferred to a polyvinyl difluoride membrane. To minimize nonspecific binding, the membrane was incubated with Blocking One (Nacalai Tesque). The membrane was washed thrice with PBST (phosphate-buffered saline (PBS) containing $0.1 \%(\mathrm{v} / \mathrm{v})$ Tween-20) and incubated with anti-C4BPA polyclonal antibodies (LifeSpan BioSciences, Inc.) for $1 \mathrm{~h}$ at room temperature. Next, the membrane was washed thrice with PBST and incubated with rabbit horseradish peroxidase (HRP)-conjugated anti-mouse immunoglobulin secondary antibody (Dako Japan) for $1 \mathrm{~h}$ at room temperature. The immunoreactive bands were visualized using Pierce Western Blotting substrate (Thermo Fisher Scientific). Precision plus protein dual-color standards (Bio-Rad Laboratories, Inc.) were used as internal references.

Immobilization of antibody to a polystyrene microtiter plate. The anti-C4BPA polyclonal antibodies in PBS were dispensed into a 96-well polystyrene microtiter plate (Thermo Fisher Scientific) at a concentration of $0.5 \mathrm{mg} /$ well and incubated for 1 day at $4^{\circ} \mathrm{C}$. The plate was washed thrice with PBS containing $0.05 \%$ Tween-20. Next, the wells of the microtiter plate were coated with $20 \%$ NOF102 containing $10 \%$ sucrose for one day at $4^{\circ} \mathrm{C}$, followed by drying for seven days at $4^{\circ} \mathrm{C}$. The microtiter plate was maintained at $4^{\circ} \mathrm{C}$ until use.

LCA-lectin ELISA conditions. First, 100- $\mu 1$ aliquots of 100 -fold diluted serum samples were added in duplicates to each well of a microtiter plate washed with PBS. The samples were incubated at room temperature for $1 \mathrm{~h}$ and washed thrice. HRP-conjugated LCA-lectin (J-Chemical, Inc.) in PBS containing $0.05 \%$ Tween-20 $(100 \mu \mathrm{l})$ was added to each well and the samples were incubated at room temperature for $30 \mathrm{~min}$. The plate was washed thrice and $100 \mu \mathrm{l}$ of TMB solution (Fujifilm Wako Pure Chemical Corporation) was added to each well. After incubation at room temperature for $10 \mathrm{~min}$, $100 \mu 1$ of stop solution was added. The absorbance of the mixtures was measured at $450 \mathrm{~nm}$.

Statistical analysis. Numerical data are presented as the means \pm standard deviations. All statistical analyses, including linear regression analysis and Pearson's correlation coefficient calculation were performed, and receiver operator characteristic (ROC) and the area under the ROC curves (AUC) were calculated using SPSS v.19.0 (SPSS). For non-parametric data, the means of the two groups were compared using Mann-Whitney U test, Welch's t-test. The means of the two groups among three groups were compared using Dunn's nonparametric comparison for post hoc testing after a Kruskal-Wallis test. The means of four groups were compared the differences using ANOVA test and the Tukey-Kramer post hoc test. The differences were considered significant at $\mathrm{P}<0.05$.

\section{Results}

Establishment and characterization of a hybrid LCA-lectin ELISA for measuring Fuc-C4BPA. We first developed a sandwich ELISA system, which has a higher sensitivity than a previously reported ELISA system (4), for measuring total C4BPA levels. The anti-C4BPA antibody was used to detect recombinant human $\mathrm{C} 4 \mathrm{BPA}$ protein and serum $\mathrm{C} 4 \mathrm{BPA}$ in a 
Table I. Clinical characteristics of participants.

\begin{tabular}{|c|c|c|c|}
\hline \multirow[b]{2}{*}{ Patient set } & \multirow[b]{2}{*}{ Age, mean \pm SD } & \multicolumn{2}{|c|}{ Sex } \\
\hline & & M & $\mathrm{F}$ \\
\hline \multicolumn{4}{|l|}{ Training set } \\
\hline HVs $(n=40)$ & $65 \pm 5$ & 25 & 15 \\
\hline $\mathrm{CP}(\mathrm{n}=10)$ & $51 \pm 14$ & 9 & 1 \\
\hline PDAC $(n=19)$ & $68 \pm 10$ & 11 & 8 \\
\hline \multicolumn{4}{|l|}{ Validation set 1} \\
\hline Pre-/post-operative sera of PDAC $(n=9)$ & $69 \pm 13$ & 5 & 4 \\
\hline \multicolumn{4}{|l|}{ Validation set 2} \\
\hline HVs $(n=10)$ & $66 \pm 5$ & 5 & 5 \\
\hline $\mathrm{CP}(\mathrm{n}=10)$ & $58 \pm 12$ & 9 & 1 \\
\hline $\operatorname{PDAC}(\mathrm{n}=17)$ & $63 \pm 11$ & 9 & 8 \\
\hline \multicolumn{4}{|l|}{ Validation set 3} \\
\hline HVs $(n=50$; training set + validation set 2$)$ & $67 \pm 5$ & 30 & 20 \\
\hline $\mathrm{CP}(\mathrm{n}=20$; training set + validation set 2$)$ & $55 \pm 13$ & 18 & 2 \\
\hline PDAC $(n=45$; training set + validation set $1+2)$ & $66 \pm 11$ & 25 & 20 \\
\hline
\end{tabular}

HVs, healthy volunteers; $\mathrm{CP}$, chronic pancreatitis; PDAC, pancreatic ductal adenocarcinoma; M, male; F, female.

patient with PDAC (Fig. 1A). This antibody was used as a capture antibody to establish a novel hybrid LCA-lectin ELISA for measuring the Fuc-C4BPA levels (Fig. 1B). A standard curve was generated based on the absorbance value of the diluted C4BPA in the HEK293T cell lysate (OriGene Technologies, Inc.) to evaluate the correlation between the absorbance values and the C4BPA concentrations. The working concentration range of Fuc-C4BPA was 0.0-2.0 AU/ml (Fig. 1C). The assay results revealed a linear correlation between the absorbance values and the C4BPA levels at a concentration range of $0.0-2.0 \mathrm{AU} / \mathrm{ml}\left(\mathrm{y}=1.0009 \mathrm{x}, \mathrm{r}^{2}=0.9988, \mathrm{P}<0.0001\right.$; Pearson's correlation) (Fig. 1D). The detection limit was estimated by assaying the zero concentration eight times and was defined as the C4BPA 'zero' concentration +3 SD. The limit of Fuc-C4BPA detection was $0.13 \mathrm{AU} / \mathrm{ml}$. To examine the within-run and between-run reproducibility, the precision of the assay was examined at Fuc-C4BPA concentrations of 0.23 and $1.39 \mathrm{AU} / \mathrm{ml}$. Within-assay coefficient of variations (CVs) were determined using eight replicates of each sample. The assay was repeated on five different days to determine the between-assay CVs (two replicates of each sample per day). The within-run and between-run CVs were 2.6-6.7 and $1.8-3.6 \%$, respectively.

Finally, interference was assessed in the samples containing $0.23 \mathrm{AU} / \mathrm{ml}$ of Fuc-C4BPA. Potential interference materials were added to the sera at various concentrations. There was no substantial interference from hemoglobin (up to $5,000 \mathrm{mg} / \mathrm{l}$ ), free bilirubin (up to $207 \mathrm{mg} / \mathrm{l}$ ), ditaurobilirubin (up to $204 \mathrm{mg} / \mathrm{l}$ ), chyle (up to 1,400 formazine turbidity units, equal to $1,176 \mathrm{mg} / 1$ triglycerides), ascorbic acid (up to $500 \mathrm{mg} / \mathrm{l}$ ), and rheumatoid factor (up to $500 \mathrm{U} / \mathrm{l}$ ).

Fucosylated C4BPA levels are upregulated in the serum of patients with PDAC. The established LCA-lectin ELISA was used to examine the serum Fuc-C4BPA levels in 69 serum samples (40 age-matched $\mathrm{HVs}, 10$ patients with $\mathrm{CP}$, and 19 patients with PDAC) of the training set (Table I). As shown in Fig. 2A, the serum level of Fuc-C4BPA in patients with PDAC $(0.61 \pm 0.33 \mathrm{AU} / \mathrm{ml})$ was significantly higher than that in HVs $(0.21 \pm 0.06 \mathrm{AU} / \mathrm{ml} ; \mathrm{P}<0.0001)$ and patients with $\mathrm{CP}$ $(0.25 \pm 0.03 \mathrm{AU} / \mathrm{ml} ; \mathrm{P}<0.0001)$. Additionally, the serum level of Fuc-C4BPA in patients with $\mathrm{CP}$ was higher than that in HVs $(\mathrm{P}<0.01)$. Next, the Fuc-C4BPA levels were measured in nine pairs of pre- and post-operative sera in the validation set 1 . Interestingly, the serum Fuc-C4BPA level significantly decreased in all nine patients after the curative operation (Fig. 2B) ( $\mathrm{P}=0.0019$; Wilcoxon signed rank test).

Fucosylated C4BPA levels have a higher PDAC detection accuracy than total C4BPA levels. To examine the discriminatory power of serum Fuc-C4BPA and total C4BPA levels, we compared the levels of Fuc-C4BPA and total C4BPA among 17 patients with PDAC and age-matched benign controls comprising $10 \mathrm{HVs}$ and 10 patients with $\mathrm{CP}$ in the validation set 2. The serum levels of total C4BPA in patients with PDAC $(87.7 \pm 41.7 \mu \mathrm{g} / \mathrm{ml})$ were significantly higher than those in HVs $(38.9 \pm 8.4 \mu \mathrm{g} / \mathrm{ml})(\mathrm{P}<0.001)$ and patients with $\mathrm{CP}$ $(43.4 \pm 11.5 \mu \mathrm{g} / \mathrm{ml})(\mathrm{P}<0.01)$. The HVs and patients with $\mathrm{CP}$ did not exhibit significant differences in the serum levels of total C4BPA ( $\mathrm{P}=0.35)$ (Fig. 3A). Meanwhile, the serum levels of Fuc-C4BPA in patients with PDAC $(0.54 \pm 0.22 \mathrm{AU} / \mathrm{ml})$ were significantly higher than those in HVs $(0.20 \pm 0.04 \mathrm{AU} / \mathrm{ml}$; $\mathrm{P}<0.0001)$ and patients with $\mathrm{CP}(0.25 \pm 0.03 \mathrm{AU} / \mathrm{ml} ; \mathrm{P}<0.0001)$ in the validation set 2 (Fig. $3 \mathrm{~B}$ ). The serum levels of total C4BPA and Fuc-C4BPA were not significantly correlated in this sample set $(\mathrm{P}=0.61, \mathrm{r}=-0.13$; Pearson's correlation) (Fig. 3C).

Next, the receiver operating characteristic (ROC) curves were generated to evaluate the ability of Fuc-C4BPA, total 
A
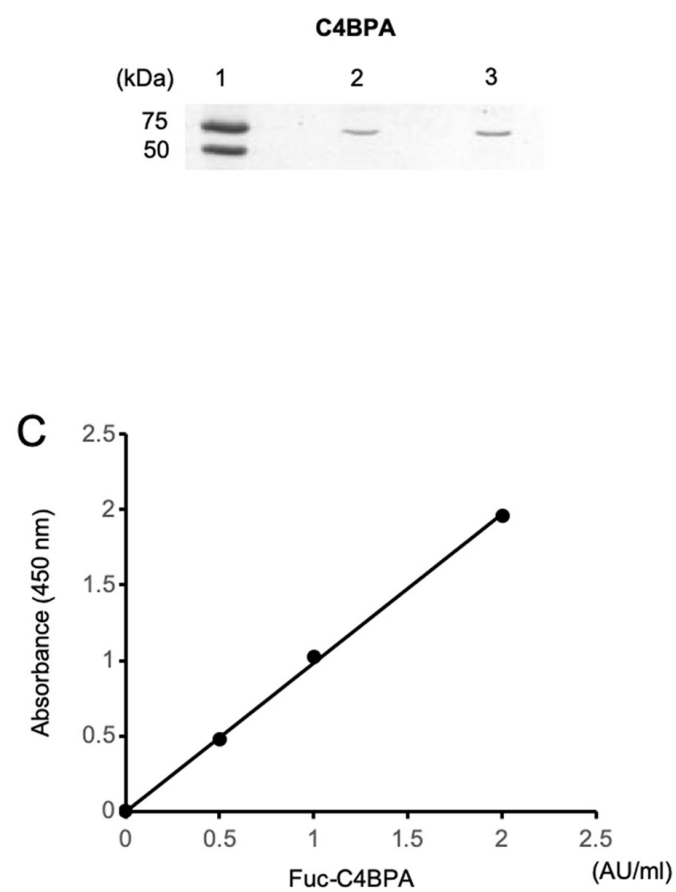

B

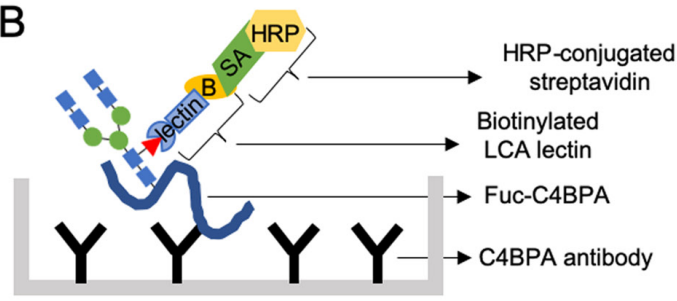

Well in the plate

$\square$ GlcNac Mannose $\triangle$ Fucose

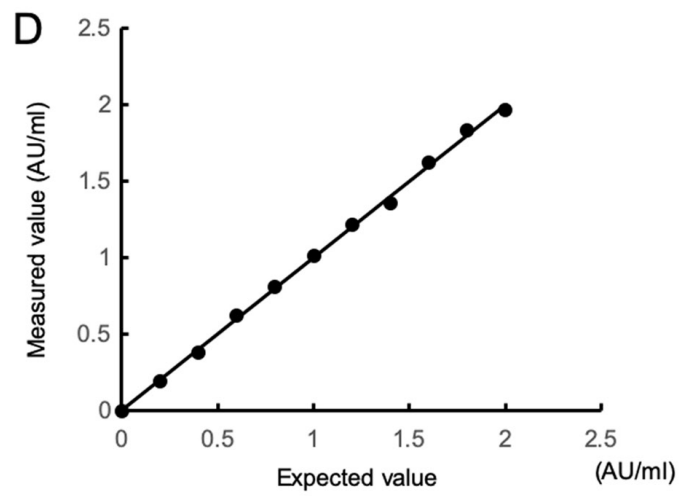

Figure 1. Establishment of lens culinaris agglutinin-lectin ELISA for the detection of fucosylated C4BPA. (A) Western blot analysis. Immunoreactive bands were observed at the predicted molecular weight of C4BPA $(67 \mathrm{kDa})$ upon incubation of recombinant human C4BPA protein and the serum sample from a patient with PDAC with anti-C4BPA polyclonal antibodies. Lane 1, internal references. The molecular weights of the upper and lower bands are 75 and $50 \mathrm{kDa}$, respectively. Lane 2, recombinant human C4BPA protein. Lane 3, serum sample from a patient with PDAC. (B) Schematic diagram of the antibody-lectin sandwich assay. Immobilized anti-C4BPA antibody captures C4BPA in the serum. Fucosylation of C4BPA is detected using a biotinylated lectin, followed by incubation with horseradish peroxidase-conjugated streptavidin. (C) Standard curves of Fuc-C4BPA following ELISA. Correlation between absorbance values and concentration of Fuc-C4BPA. Four concentrations of Fuc-C4BPA were examined using ELISA. (D) Linearity of the ELISA results, which fitted into the following linear equation: $\mathrm{y}=1.0009 \mathrm{x}\left(\mathrm{r}^{2}=0.9988\right.$; $\left.\mathrm{P}<0.0001\right)$. C4BPA, C4b-binding protein $\alpha$-chain; PDAC, pancreatic ductal adenocarcinoma; HRP, horseradish peroxidase; GlcNac, N-Acetylglucosamine; Fuc, fucosylated; LCA, lens culinaris agglutinin.
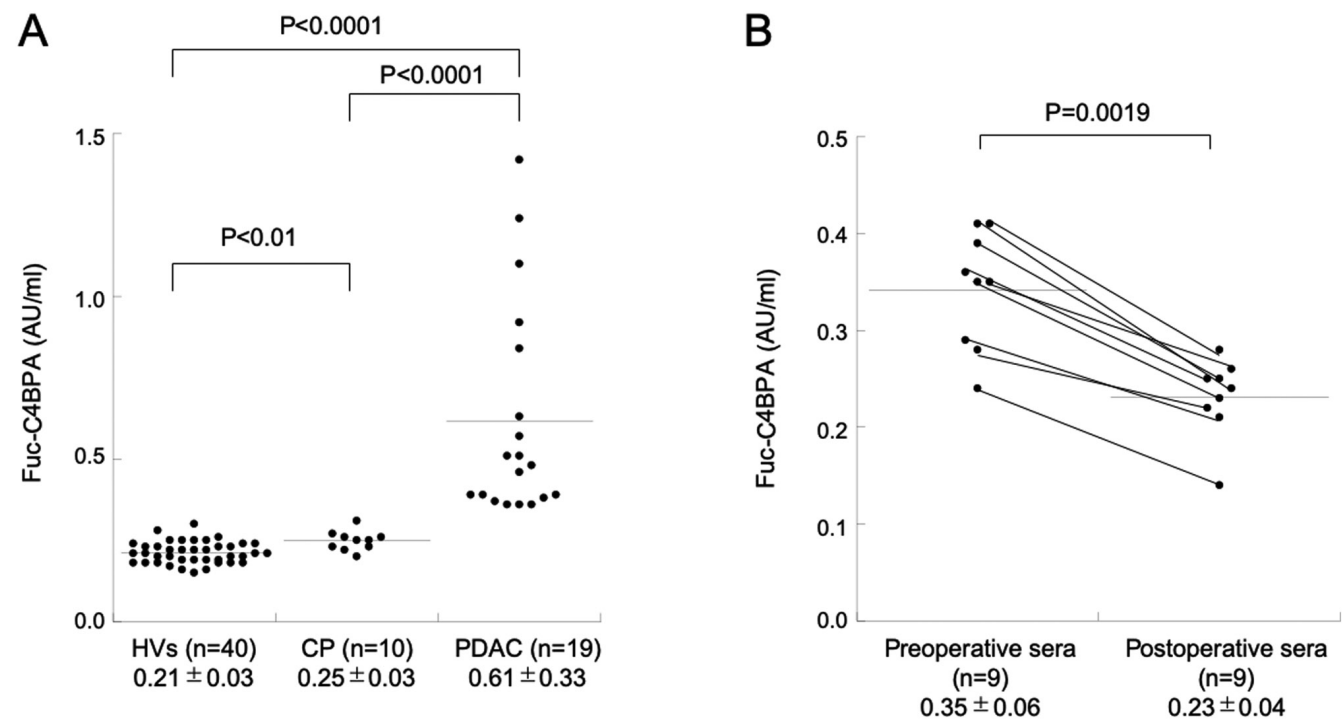

Figure 2. Serum Fuc-C4BPA levels are upregulated in patients with PDAC. (A) Comparison of serum Fuc-C4BPA levels between HVs, patients with CP and patients with PDAC using ELISA. Serum Fuc-C4BPA levels in patients with PDAC were significantly higher than those in HVs and patients with CP. (B) Comparison of serum Fuc-C4BPA levels before and after surgery in patients with PDAC. Fuc-C4BPA, fucosylated C4b-binding protein $\alpha$-chain; PDAC, pancreatic ductal adenocarcinoma; $\mathrm{HV}$, healthy volunteers; $\mathrm{CP}$, chronic pancreatitis.

C4BPA, CA19-9, and CEA levels to distinguish patients with PDAC from non-cancer (HVs and CP) participants. The cutoff values of these four markers were set at levels (mean $+2 \mathrm{SD}$ ) that yielded a specificity of $97.7 \%$ when compared with those of the participants without cancer ( $\mathrm{HVs}$ and $\mathrm{CP}$ patients); $61.3 \mu \mathrm{g} / \mathrm{ml}, 0.297 \mathrm{AU} / \mathrm{ml}, 34.2 \mathrm{U} / \mathrm{ml}$, and $7.3 \mathrm{ng} / \mathrm{ml}$ for total 
A
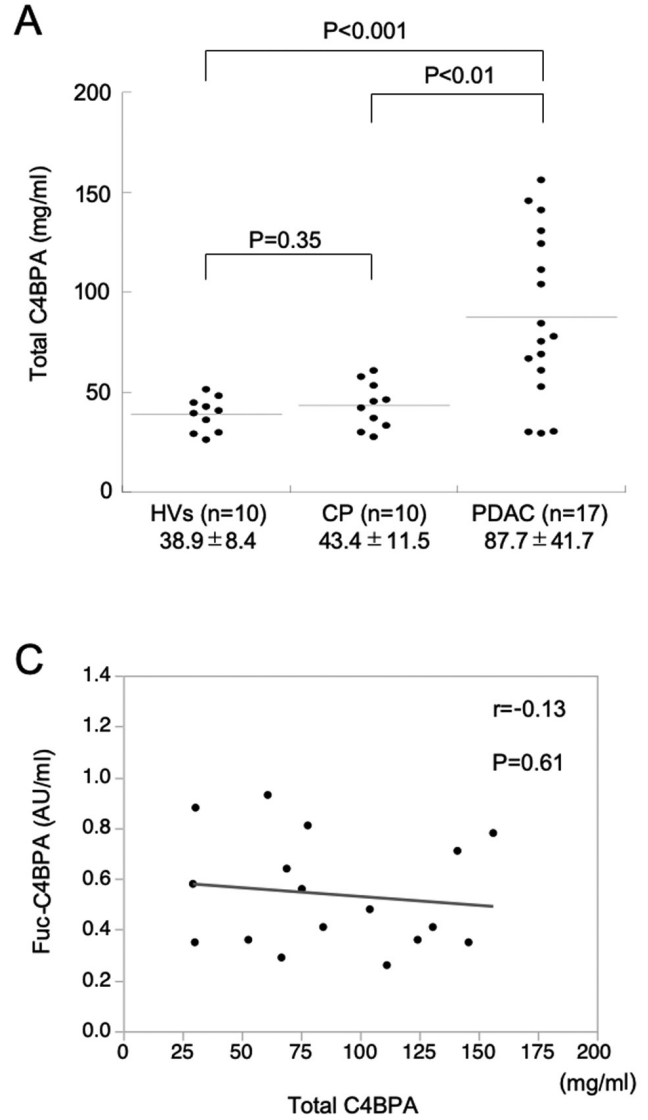

B
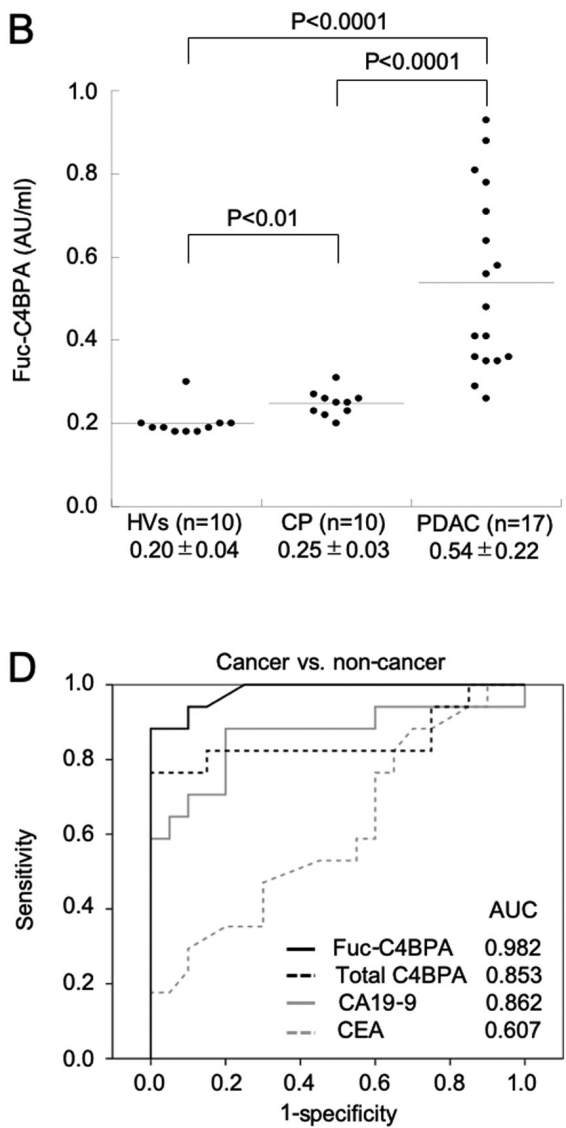

Figure 3. Serum levels of total C4BPA and Fuc-C4BPA are upregulated in patients with PDAC. (A) Comparison of total serum C4BPA levels among HVs, patients with CP and patients with PDAC using ELISA. Total serum C4BPA levels in patients with PDAC were significantly higher than those in HVs and patients with CP. HVs and patients with CP did not exhibit significant differences in total serum C4BPA levels. (B) Comparison of Fuc-C4BPA serum levels between HVs, patients with CP and patients with PDAC using ELISA. The serum Fuc-C4BPA levels in patients with PDAC were significantly higher than those in HVs and patients with CP. (C) Comparison of serum levels of total C4BPA and Fuc-C4BPA. Serum Fuc-C4BPA levels were not correlated with total serum C4BPA levels in patients with PDAC. (D) Fuc-C4BPA has higher PDAC diagnostic accuracy than total C4BPA, CA19-9 and carcinoembryonic antigen (CEA). The receiver operating characteristic curve analyses of the serum levels of Fuc-C4BPA, total C4BPA, CA19-9 and CEA between patients with PDAC, HVs and patients with CP. PDAC, pancreatic ductal adenocarcinoma; C4BPA, C4b-binding protein $\alpha$-chain; Fuc, fucosylated; HVs, healthy volunteers; CP, chronic pancreatitis; CA19-9, carbohydrate antigen 19-9; CEA, carcinoembryonic antigen; AUC, area under the curve.

C4BPA, Fuc-C4BPA, CA19-9, and CEA, respectively. The area under the ROC curve (AUC) of Fuc-C4BPA, total C4BPA, CA19-9, and CEA was 0.982, 0.853, 0.862, and 0.607, respectively, in the validation set 2 (Fig. 3D). These results suggest that serum Fuc-C4BPA expression is a potential novel diagnostic biomarker for PDAC.

Fuc-C4BPA can identify the CA19-9 false-negative cases among patients with PDAC. To validate the diagnostic value of serum Fuc-C4BPA levels in PDAC, the PDAC sample size in the validation set 3 was increased to 45 from the training set and the validation sets 1 and 2. The Fuc-C4BPA levels in patients with PDAC $(0.62 \pm 0.33 \mathrm{AU} / \mathrm{ml})$ were significantly higher than those in HVs $(0.21 \pm 0.03 \mathrm{AU} / \mathrm{ml} ; \mathrm{P}<0.0001)$ and patients with $\mathrm{CP}(0.25 \pm 0.03 \mathrm{AU} / \mathrm{ml} ; \mathrm{P}<0.0001)$ (Fig. 4A). The AUC of Fuc-C4BPA and CA19-9 was 0.985 and 0.904 , respectively. This indicated that serum Fuc-C4BPA is a better diagnostic PDAC biomarker than serum CA19-9 (Fig. 4B; $\mathrm{P}<0.001)$.

The accuracy of a single serum biomarker is limited in cancer detection. To increase the detection rate, additional biomarkers are often tested in a clinical setting. CA19-9 reacts with a monoclonal antibody directed against sialyl Lewis A antigen. Hence, Lewis A antigen-negative patients do not exhibit increased levels of CA19-9 (12). To overcome this limitation, the level of duke pancreatic monoclonal antigen type 2 (DUPAN-2) is measured because it is recognized by a monoclonal antibody directed against sialyl Lewis $\mathrm{C}$ antigen, which is the precursor of sialyl Lewis A antigen (13). We examined the ability of Fuc-C4BPA to identify the CA19-9false-negative cases among patients with PDAC. Among the 16 CA19-9-negative patients, the percentage of the Fuc-C4BPA-positive cases $(12 / 16 ; 75 \%)$ was significantly higher than that of DUPAN-2-positive cases $(3 / 16 ; 18.8 \%)$ ( $\mathrm{P}=0.0015$; Chi-square test) (Fig. 4C). These results indicated that Fuc-C4BPA can identify the CA19-9 false-negative cases among patients with PDAC.

Fucosylated C4BPA predicts pathological lymph node (LN) metastasis in patients with PDAC. Next, we investigated the correlation between the levels of Fuc-C4BPA and the clinicopathological features of patients with PDAC. Among the major clinical parameters, such as factors contributing to the TNM classification system of malignant tumors, the 
Table II. Fuc-C4BPA, CA19-9 and CEA levels in the sera of participants.

\begin{tabular}{lcccccccc}
\hline & \multicolumn{2}{c}{ Non-cancer $(\mathrm{n}=70)$} & & \multicolumn{5}{c}{ PDAC (validation set 3; $\mathrm{n}=45)$} \\
\cline { 2 - 3 } Serum markers & HVs $(\mathrm{n}=50)$ & $\mathrm{CP}(\mathrm{n}=20)$ & & Stage I $(\mathrm{n}=8)$ & Stage II $(\mathrm{n}=14)$ & Stage III $(\mathrm{n}=19)$ & Stage IV $(\mathrm{n}=4)$ & P-value \\
\hline Fuc-C4BPA $(\mathrm{AU} / \mathrm{ml})$ & $0.21 \pm 0.03$ & $0.25 \pm 0.03$ & & $0.38 \pm 0.05$ & $0.57 \pm 0.29$ & $0.58 \pm 0.29$ & $0.48 \pm 0.17$ & 0.13 \\
CA19-9 $(\mathrm{U} / \mathrm{ml})$ & $11.2 \pm 8.1$ & $16.7 \pm 14.5$ & & $1,697.5 \pm 4,301.4$ & $208.8 \pm 398.8$ & $365.2 \pm 467.5$ & $347.6 \pm 317.7$ & 0.65 \\
CEA $(\mathrm{ng} / \mathrm{ml})$ & $3.1 \pm 1.9$ & $3.5 \pm 2.4$ & & $10.8 \pm 18.6$ & $4.6 \pm 3.6$ & $3.1 \pm 2.5$ & $3.0 \pm 1.7$ & 0.47 \\
\hline
\end{tabular}

Data are presented as the mean \pm SD. PDAC staging was performed using the 8th edition of TNM Classification of malignant tumors. HVs, healthy volunteers; $\mathrm{CP}$, chronic pancreatitis; PDAC, pancreatic ductal adenocarcinoma; Fuc-C4BPA, fucosylated C4b-binding protein $\alpha$-chain; CA19-9, carbohydrate antigen 19-9; CEA, carcinoembryonic antigen.

A
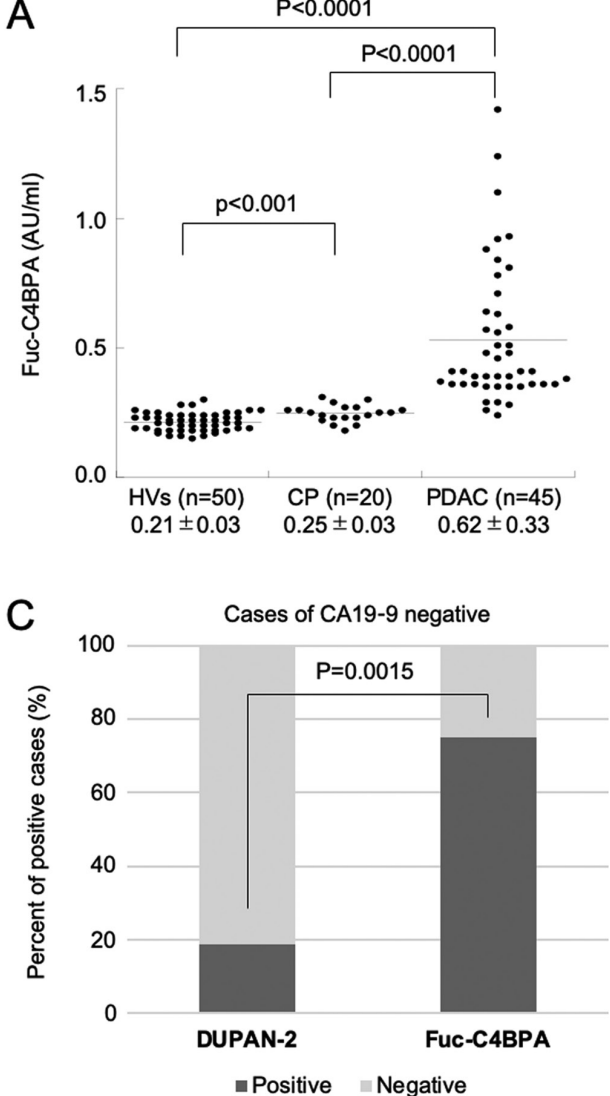

B

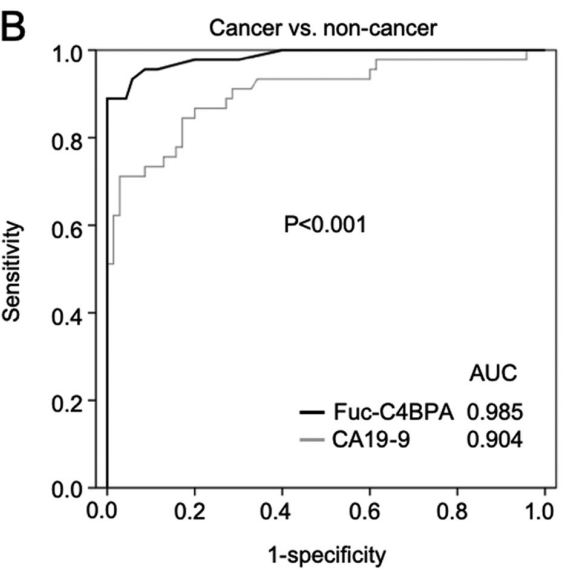

Figure 4. Confirmation of upregulated serum Fuc-C4BPA levels in patients with PDAC in the validation set 3. (A) Comparison of serum Fuc-C4BPA levels among HVs, patients with CP and patients with PDAC using ELISA. Serum Fuc-C4BPA levels in patients with PDAC were significantly higher than those in HVs and patients with CP. (B) Fuc-C4BPA has increased PDAC diagnostic accuracy compared with CA19-9. The receiving operating characteristic curve analyses of Fuc-C4BPA and CA19-9 serum levels in patients with PDAC, HVs and patients with CP. (C) The ability of Fuc-C4BPA to identify CA19-9-negative cases among patients with PDAC. The percentage of the Fuc-C4BPA-positive cases (75\%) was significantly higher than that of duke pancreatic monoclonal antigen type 2-positive cases (18.8\%) among CA 19-9-negative patients with PDAC. Fuc-C4BPA, fucosylated C4b-binding protein $\alpha$-chain; PDAC, pancreatic ductal adenocarcinoma; HVs, healthy volunteers; CP, chronic pancreatitis; DUPAN-2, duke pancreatic monoclonal antigen type 2; AUC, area under the curve.

tumor stage was not correlated with the levels of Fuc-C4BPA and CA19-9 (Table II). Pathological LN metastasis ( $\mathrm{pN}+$ ) was positively correlated with high serum Fuc-C4BPA levels in the validation set 3 ( $\mathrm{P}=0.035$; Welch's t-test) (Fig. 5A). Furthermore, the ability of Fuc-C4BPA and CA19-9 levels to predict LN metastasis was comparatively evaluated. The AUC of serum Fuc-C4BPA levels (0.703) was significantly higher than that of serum CA19-9 levels $(0.500)(\mathrm{P}<0.001)$ (Fig. 5B). These findings suggested that Fuc-C4BPA is a better predictor of LN metastasis than CA19-9 in patients with PDAC.

\section{Discussion}

Although several novel biomarkers have been identified using proteomic analysis, most of them are not available for clinical diagnosis. Generally, biomarkers are identified through comparative analysis of the disease and control groups based 
A

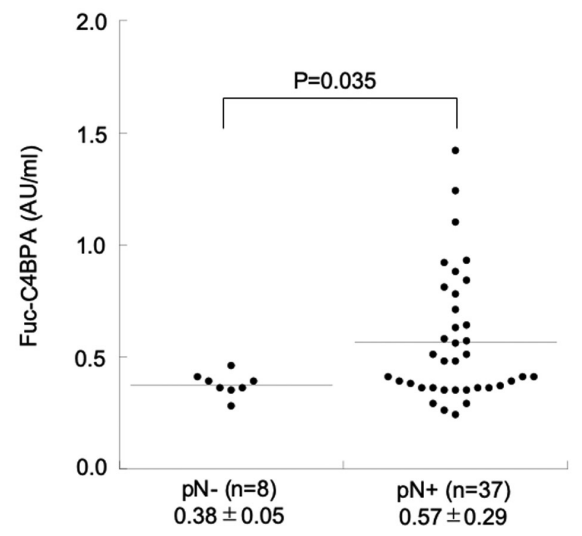

B

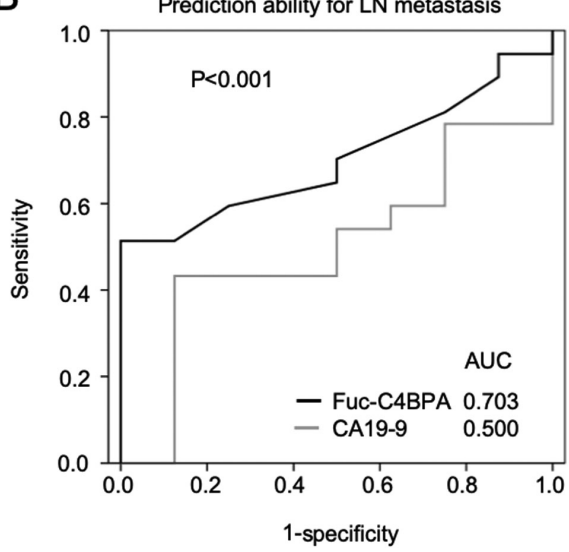

Figure 5. Correlation between serum Fuc-C4BPA levels and lymph node metastasis in patients with pancreatic ductal adenocarcinoma. (A) Patients with lymph node metastasis exhibited significantly higher serum Fuc-C4BPA levels than patients without lymph node metastasis in the validation set 3. (B) The area under the curve of serum Fuc-C4BPA levels (0.703) was significantly higher than that of serum carbohydrate antigen 19-9 levels (0.500), which indicated the ability of Fc-C4BPA to predict lymph node metastasis. Fuc-C4BPA, fucosylated C4b-binding protein $\alpha$-chain; pN+, patients with lymph node metastasis; PN-, patients without lymph node metastasis; LN, lymph node; AUC, area under the curve; CA19-9, carbon antigen 19-9.

on semiquantitative methods (discovery phase) and identification of candidates using MS (validation phase). Multiple reaction monitoring (MRM) in MS, which does not involve the use of an antibody, and selective reaction monitoring are used to detect and quantify the target proteins (11). The serum Fuc-C4BPA level cannot be measured using MRM as its molecular weight exceeds the detectable range of MRM. Clinically, steroid hormones (14), vitamins (15), and thyroid hormones (16) can be measured using MS. However, the clinical applications of MS for diagnosis are limited due to various limitations, including complex sample preparation, lack of high-throughput methods, and the need for automation, interface to the laboratory information system, and device standardization. Previously, the serum level of fully-sialylated C4BPA was reported to be upregulated in patients with clear-cell ovarian cancer. However, the utility of the serum level of fully-sialylated C4BPA for cancer diagnosis has been limited due to the absence of a high-throughput method for measurement (10).

Cancer progression encompasses major alterations in molecular glycosylation, such as incomplete synthesis of $\mathrm{O}$-glycan structures, increased expression of branched $\mathrm{N}$-glycans, expression of terminal sialylated glycans, and altered fucosylation. Capturing lectin using Aleuria aurantia lectin, Pisum sativum agglutinin, and LCA, which can recognize the core fucosylation structure, is the established approach to identify the core fucosylated glycoproteins. In this study, we developed an ELISA using C4BPA polyclonal antibodies and LCA-lectin. The ELISA system exhibited good basic performances, including day-to-day reproducibility and repeatability. The easy-to-use ELISA system developed in this study to detect serum Fuc-C4BPA is a useful method for diagnosing PDAC.

Cancer-related immunocompetent cells or complement factors in the tumor microenvironment are associated with cancer progression (17). The contribution of aberrant fucosylation to the interactions within the tumor microenvironment remains poorly understood. In this study, we demonstrated that the serum Fuc-C4BPA levels were significantly upregulated in patients with PDAC. Additionally, the serum Fuc-C4BPA levels in the post-operative patients were significantly lower than those in the preoperative patients. Fucosylation is reported to be upregulated at an early stage of colon carcinogenesis (18). However, other studies have reported that de-fucosylation through genetic mutation promotes the escape from natural killer cell-mediated tumor surveillance and the development of malignant characteristics in certain types of advanced cancer (19). To understand the role of fucosylation during cancer progression from early to late-stage, the mechanisms underlying fucosylation or de-fucosylation induced by the interaction between tumor cells and molecules in the tumor microenvironments must be elucidated.

The upregulated CA 19-9 levels are reported to be an independent prognostic factor for LN metastasis. Preoperative chemotherapy can improve the prognosis of patients with PDAC exhibiting LN metastasis $(20,21)$. Therefore, the prediction of LN metastasis is important to develop an efficient therapeutic strategy for patients with PDAC. Contrast-enhanced computed tomography (CECT) is commonly used for the preoperative diagnosis of $\mathrm{LN}$ metastasis in patients with PDAC. A previous prospective study has demonstrated that the diagnostic accuracy of LN metastasis through CECT is low $(73 \%)$ in PDAC (22). The significance of enlarged LNs in PDAC is not well defined because the LNs can enlarge due to local inflammation or biliary obstruction. Additionally, $\mathrm{LN}$ metastasis is not correlated with this enlargement (23). A recent study reported that a six-microRNA risk prediction model could distinguish patients with PDAC patients exhibiting LN metastases from those with PDAC not exhibiting LN metastases (AUCof 0.84 and 0.73 in the training and validation sets, respectively) (24). In this study, the upregulated levels of Fuc-C4BPA were positively correlated with LN metastasis. The AUC of Fuc-C4BPA (0.703) was significantly higher than that of CA19-9 (0.500). This indicated that a single serum biomarker, Fuc-C4BPA, is a good indicator of LN metastasis and can aid in determining the treatment strategy for patients with PDAC. 
There are several limitations associated with the clinical application of serum Fuc-C4BPA level as a diagnostic marker for PDAC. The diagnostic window (e.g. the detection limit) of the lectin ELISA for measuring the Fuc-C4BPA level is narrow. Additionally, the serum Fuc-C4BPA level, but not the serum total C4BPA level, in patients with $\mathrm{CP}$ was significantly higher than that in HVs. This may be due to the correlation between inflammation and carcinogenesis, which are critical for the activation of various common molecules. The identification and measurement of specific target sites of fucosylation, which are directly involved in PDAC progression, may resolve these issues. Moreover, this is a retrospective study involving a small sample size. Further validation in an independent and prospective large cohort is needed to establish Fuc-C4BPA as a promising serum diagnostic biomarker for PDAC. In conclusion, we established a novel lectin ELISA for the measurement of serum Fuc-C4BPA. Serum Fuc-C4BPA has a powerful diagnostic ability with potential applications for the development of therapeutic strategies for PDAC.

\section{Acknowledgements}

Not applicable.

\section{Funding}

This work was supported by a Grant-in-Aid for Scientific Research (KAKENHI; grant nos. 'KIBAN' C:19K07947, 19K09113, 20K09073 and 'KIBAN' B:19H03725).

\section{Availability of data and materials}

The datasets used and/or analyzed during the current study are available from the corresponding author on reasonable request.

\section{Authors' contributions}

$\mathrm{KaS}, \mathrm{SY}, \mathrm{ST}, \mathrm{FN}$, and MO were involved in the study design, data analysis and development of the study. KoS, YM, HT KF, TT, and SK collected and analyzed the clinical data. All authors read and approved the final manuscript.

\section{Ethics approval and consent to participate}

The present study was approved by the Ethics Committee of Chiba University, Graduate School of Medicine (approval no. \#2155) and Kashiwado Hospital (approval no. \#007), respectively. Informed consent was obtained from all participants and patients for the acquisition of clinical and pathological information and for the use of serum samples.

\section{Patient consent for publication}

Not applicable.

\section{Competing interests}

The authors declare that they have no competing interests.

\section{References}

1. Takano S, Yoshitomi H, Togawa A, Sogawa K, Shida T, Kimura F, Shimizu H, Tomonaga T, Nomura F and Miyazaki M: Apolipoprotein C-1 maintains cell survival by preventing from apoptosis in pancreatic cancer cells. Oncogene 27: 2810-2822, 2008.

2. Moulder R, Bhosale SD, Goodlett DR and Lahesmaa R: Analysis of the plasma proteome using iTRAQ and TMT-based Isobaric labeling. Mass Spectrom Rev 37: 583-606, 2018.

3. Bhawal R, Oberg AL, Zhang S and Kohli M: Challenges and opportunities in clinical applications of blood-based proteomics in cancer. Cancers (Basel) 12: 2428, 2020.

4. Sogawa K, Takano S, Iida F, Satoh M, Tsuchida S, Kawashima Y, Yoshitomi H, Sanda A, Kodera Y, Takizawa H, et al: Identification of a novel serum biomarker for pancreatic cancer, $\mathrm{C} 4 \mathrm{~b}$-binding protein $\alpha$-chain (C4BPA) by quantitative proteomic analysis using tandem mass tags. Br J Cancer 115: 949-956, 2016.

5. Mikami M, Tanabe K, Matsuo K, Miyazaki Y, Miyazawa M, Hayashi M, Asai S, Ikeda M, Shida M, Hirasawa T, et al: Fully-sialylated alpha-chain of complement 4-binding protein: Diagnostic utility for ovarian clear cell carcinoma. Gynecol Oncol 139: 520-528, 2015.

6. Liu YS, Luo XY, Li QR, Li H, Li C, Ni H, Li RX, Wang R, $\mathrm{Hu} \mathrm{HC}$, Pan YJ, et al: Shotgun and targeted proteomics reveal that pre-surgery serum levels of LRG1, SAA, and C4BP may refine prognosis of resected squamous cell lung cancer. J Mol Cell Biol 4: 344-347, 2012.

7. Jia L, Zhang J, Ma T, Guo Y, Yu Y and Cui J: The function of fucosylation in progression of lung cancer. Front Oncol 8: 565, 2018.

8. Li D and Satomura S: Biomarkers for hepatocellular carcinoma (HCC): An update. Adv Exp Med Biol 867: 179-193, 2015.

9. Jelski W and Mroczko B: Biochemical diagnostics of pancreatic cancer-Present and future. Clin Chim Acta 498: 47-51, 2019.

10. Tanabe K, Matsuo K, Miyazawa M, Hayashi M, Ikeda M, Shida M, Hirasawa T, Sho R and Mikami M: UPLC-MS/MS based diagnostics for epithelial ovarian cancer using fully sialylated C4-binding protein. Biomed Chromatogr 32: e4180, 2018.

11. Umemura H, Nezu M, Kodera Y, Satoh M, Kimura A, Tomonaga T and Nomura F: Effects of the time intervals between venipuncture and serum preparation for serum peptidome analysis by matrix-assisted laser desorption/ionization time-of-flight mass spectrometry. Clin Chim Acta 406: 179-180, 2009.

12. Narimatsu H, Iwasaki H, Nakayama F, Ikehara Y, Kudo T, Nishihara S, Sugano K, Okura H, Fujita S and Hirohashi S: Lewis and secretor gene dosages affect CA19-9 and DU-PAN-2 serum levels in normal individuals and colorectal cancer patients. Cancer Res 58: 512-518, 1998.

13. Kawa S, Oguchi H, Kobayashi T, Tokoo M, Furuta S, Kanai M and Homma T: Elevated serum levels of Dupan-2 in pancreatic cancer patients negative for Lewis blood group phenotype. Br J Cancer 64: 899-902, 1991.

14. Stanczyk FZ and Clarke NJ: Advantages and challenges of mass spectrometry assays for steroid hormones. J Steroid Biochem Mol Biol 121: 491-495, 2010.

15. Farrell CJ, Martin S, McWhinney B, Straub I, Williams P and Herrmann M: State-of-the-art vitamin D assays: A comparison of automated immunoassays with liquid chromatography-tandem mass spectrometry methods. Clin Chem 58: 531-542, 2012.

16. Soldin SJ, Soukhova N, Janicic N, Jonklaas J and Soldin OP: The measurement of free thyroxine by isotope dilution tandem mass spectrometry. Clin Chim Acta 358: 113-118, 2005.

17. Reis ES, Mastellos DC, Ricklin D, Mantovani A and Lambris JD: Complement in cancer: Untangling an intricate relationship. Nat Rev Immunol 18: 15-18, 2018.

18. Muinelo-Romay L, Vazquez-Martin C, Villar-Portela S, Cuevas E, Gil-Martín E and Fernández-Briera A: Expression and enzyme activity of alpha $(1,6)$ fucosyltransferase in human colorectal cancer. Int J Cancer 123: 641-646, 2008.

19. Moriwaki K, Noda K, Furukawa Y, Ohshima K, Uchiyama A, Nakagawa T, Taniguchi N, Daigo Y, Nakamura Y, Hayashi N and Miyoshi E: Deficiency of GMDS leads to escape from NK cell-mediated tumor surveillance through modulation of TRAIL signaling. Gastroenterology 137: 188-198, 2009.

20. Lambert A, Schwarz L, Borbath I, Henry A, Van Laethem JL, Malka D, Ducreux M and Conroy T: An update on treatment options for pancreatic adenocarcinoma. Ther Adv Med Oncol 11: 1758835919875568, 2019. 
21. Tran Cao HS, Zhang Q, Sada YH, Silberfein EJ, Hsu C, Van Buren G II, Chai C, Katz MHG, Fisher WE and Massarweh NN: Value of lymph node positivity in treatment planning for early stage pancreatic cancer. Surgery 162: 557-567, 2017.

22. Roche CJ, Hughes ML, Garvey CJ, Campbell F, White DA, Jones L and Neoptolemos JP: CT and pathologic assessment of prospective nodal staging in patients with ductal adenocarcinoma of the head of the pancreas. AJR Am J Roentgenol 180 475-480, 2003

23. Tseng DS, van Santvoort HC, Fegrachi S, Besselink MG, Zuithoff NP, Borel Rinkes IH, van Leeuwen MS and Molenaar IQ: Diagnostic accuracy of CT in assessing extra-regional lymphadenopathy in pancreatic and peri-ampullary cancer: A systematic review and meta-analysis. Surg Oncol 23: 229-235, 2014.
24. Nishiwada S, Sho M, Banwait JK, Yamamura K, Akahori T, Nakamura K, Baba $\mathrm{H}$ and Goel A: A microRNA signature identifies pancreatic ductal adenocarcinoma patients at risk for lymph node metastases. Gastroenterology 159: 562-574, 2020.

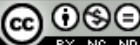

$$
\begin{aligned}
& \text { This work is licensed under a Creative Commons } \\
& \text { Attribution-NonCommercial-NoDerivatives } 4.0 \\
& \text { International (CC BY-NC-ND 4.0) License. }
\end{aligned}
$$

